\title{
Peculiarities of $\alpha$-element Abundances in Galactic Open Clusters
}

\author{
V. A. Marsakov, M. L.Gozha, V. V. Koval', L. V. Shpigel' \\ Southern Federal University, Rostov-on-Don, Russia \\ e-mail: marsakov@sfedu.ru,vvkoval@sfedu.ru \\ accepted 2016, Astronomy Reports, Vol. 60, No. 1, pp. 43-60
}

\begin{abstract}
A catalog compiling the parameters of 346 open clusters, including their metallicities, positions, ages, and velocities has been composed. The elements of the Galactic orbits for 272 of the clusters have been calculated. Spectroscopic determinations of the relative abundances, $[\mathrm{el} / \mathrm{Fe}]$, for 14 elements synthesized in various nuclear processes averaged over data from 109 publications are presented for 90 clusters. The compiled data indicate that the relative abundances of primary $\alpha$ elements (oxygen and magnesium) exhibit different dependences on metallicity, age, Galactocentric distance, and the elements of the Galactic orbits in clusters with high, elongated orbits satisfying the criterion $\left(Z_{\max }^{2}+4 e^{2}\right)^{1 / 2}>0.40$ and in field stars of the Galactic thin disk $\left(Z_{\max }\right.$ is the maximum distance of the orbit from the Galactic plane in kiloparsec and $e$ is the eccentricity of the Galactic orbit). Since no systematic effects distorting the relative abundances of the studied elements in these clusters have been found, these difference suggest real differences between clusters with high, elongated orbits and field stars. In particular, this supports the earlier conclusion, based on an analysis of the elements of the Galactic orbits, that some clusters formed as a result of interactions between high-velocity, metal-poor clouds and the interstellar medium of the Galactic thin disk. On average, clusterswith high, elongated orbits and metallicities $[\mathrm{Fe} / \mathrm{H}]<-0.1$ display lower relative abundances of the primary elements than do field stars. The low $[\mathrm{O}, \mathrm{Mg} / \mathrm{Fe}]$ ratios of these clusters can be understood if the high-velocity clouds that gave rise to them were formed of interstellar material from regions where the star-formation rate and/or the masses of Type II supernovae were lower than near the Galactic plane. It is also shown that, on average, the relative abundances of the primary elements are higher in relatively metal-rich clusters with high, elongated orbits than in field stars. This can be understood if clusters with $[\mathrm{Fe} / \mathrm{H}]>-0.1$ formed as a result of interactions between metal-rich clouds with intermediate velocities and the interstellar medium of the Galactic disk; such clouds could form from returning gas in a so-called "Galactic fountain".
\end{abstract}

Key words: open star clusters, chemical composition, kinematics, Galaxy (Milky Way).

\section{Introduction}

The relative elemental abundances $[\mathrm{el} / \mathrm{Fe}]$ in stellar atmospheres carry information about the chemical evolution of the interstellar material from which the stars were formed. This is true because different nuclear-synthesis processes occur in stars of different masses, which evolve at different rates, and so eject the heavy elements they produce into the interstellar medium on different time scales. As a result, the relative elemental abundances in the atmospheres of subsequent generations of stars with various ages can be used to trace the history of the enrichment of the stellar-gas system in various chemical elements.

As typical representatives of the Galactic thin disk, open clusters are often used to analyze the spatial structure and chemical properties of the thin disk. However, studies of the elements of their Galactic orbits and their metallicities suggest that the population of open clusters is not homogeneous, 
and includes clusters formed as a result of interactions of high-velocity, massive clouds with interstellar material in the thin disk, and also clusters formed from interstellar material that has fallen onto the disk from outer regions of the Galaxy (see [1-[4] and references therein). This same conclusion was drawn in connection with the detection of clusters with very elongated orbits extending far from the Galactic plane having low metallicities characteristic of older Galactic subsystems the thick disk and even the halo. The possibility of forming open clusters with high, non-circular orbits and low metallicities as a result of this mechanism for initiating star formation has been demonstrated and theoretically modeled (see, e.g., [5]).

The chemical composition of high-velocity clouds differs from that of the interstellar matter in the thin disk, making it of interest to compare the the relative elemental abundances of clusters and field stars, which could differ. It is also important to trace possible differences in the chemical compositions of clusters with high velocities or low metallicities, uncharacteristic of thin-disk stars. Prompted by their interest in the detailed chemical compositions of clusters with different spatial and kinematic properties, Jacobson et al. 6] compared the relative abundances of three $\alpha$-elements in 12 open clusters of the outer disk, at distances from the Galactic plane $|z|>1 \mathrm{kpc}$, and 11 clusters located closer to the plane, but found no appreciable systematic differences. No evidence was found in the chemical compositions of the cluster stars in the outer disk that they were members of the thick disk. Moreover, it was noted that two clusters associated kinematically with the remnants of a dwarf elliptical galaxy in Sagittarius (Sgr DSph) [7 likewise do not display appreciable differences in the relative abundances of the studied elements for stars of the field and the thick and thin Galactic disks. Unfortunately, as is also true of other similar studies, the sample of clusters in this study is too small to enable statistically significant detections of systematic differences, which are unlikely to exceed the uncertainties in the elemental abundances in individual clusters.

The aim of the current study is a comparative analysis of the dependences of the relative abundances of $\alpha$-elements on metallicity, age, Galactocentric distance, and the parameters of the Galactic orbits, for both open clusters and field stars of the thin disk, and also for different groups of opencluster populations. In order to estimate the statistical reliability of the results, we must first collect the most accurate published spectroscopic measurements of the abundances of elements produced in various nuclear-synthesis processes in stars located in as large a selection of open clusters as possible.

\section{INPUT DATA}

We used version 3.4 (May 2014) of the catalog [8], which contains data for 2167 open clusters, for 918 of which proper motions and radial velocities are available. This version of the catalog is more than $50 \%$ larger than the version we used in our earlier studies [3, 4]. The Galactic coordinates, distances, proper motions, and radial velocities were used to calculate the Cartesian and cylinrical components of the cluster velocities. We then used these data to calculate the elements of the Galactic orbits of the clusters. The motion of the Sun relative to the Local Standard of Rest (LSR) was taken to be $(U, V, W)_{\odot}=(11.1,12.24,7.25) \mathrm{km} / \mathrm{s}$ [9], the solar Galactocentric distance to be $8.0 \mathrm{kpc}$, and the rotational speed of the LSR to be $220 \mathrm{~km} / \mathrm{s}$. The orbits were calculated for a standard model of the Galaxy having rotational symmetry [10], consisting of a central region having two spherical components with a total mass of $1.9 * 10^{10} M_{\odot}$, a disk with mass $3.842 * 10^{10} M_{\odot}$ consisting of three Miyamoto-Nagai potentials and three terms in the Miyamoto-Nagai potential imitating a thin layer of gas in the disk, and a spherical halo described by a logarithmic potential. The integration was carried out over a time interval of $10^{9}$ years using a fourth-order Runge-Kutta method that conserves energy and angular momentum. This yielded the apogalactic $R_{a}$ and perigalactic $R_{p}$ distances, the maximum distance of points on the orbit from the Galactic plane $Z_{\max }$, and the orbital eccentricity $e=\left(R_{a}-R_{p}\right) /\left(R_{a}+R_{p}\right)$. These parameters were calculated as their maximum values during the $10^{9}$ year interval. (See [8] for a discussion of the uncertainties in the estimates of the ages, distances, velocities, and Galactic orbital elements of the clusters.)

The main sources of metallicities were data from the catalogs of Heiter et al. [1] and Dias et al. 
[8] [version 3.4, May 2014]. Since Heiter et al. [11] rigorously selected the data used and averaged the metallicities over several measurements obtained using high-resolution spectra, their study yielded high-quality data. It is primarily these metallicities that we give in our table. However, for six of the clusters from [11], new data became available after the publication of this catalog. We obtained the metallicities of five of these (Collinder 110, Collinder 261, NGC 2477, NGC 2506, NGC 5822) by averaging the data of [11] and [12]. For another cluster (NGC 1901), we calculated the mean metallicity for the data of [8, 11] (the metallicity for this cluster in [8] was determined in 2014). The metallicities in [8] were collected from various sources, and the $[\mathrm{Fe} / \mathrm{H}]$ values were determined using both spectroscopic and photometric methods. We added all spectroscopic values from [8] to our sample, as well as photometric $[\mathrm{Fe} / \mathrm{H}]$ measurements obtained after 2010. Moreover, we took the meanweighted photometric metallicities of [13], and then added all values that fell into only one of the lists. The photometric metallicity of Berkeley 60 determined before 2010 was obtained by averaging the data from these two catalogs. We adopted spectroscopic metallicities from [14, 16] and photometric determinations from [17] for four clusters. In all, we have collected metallicities for 346 clusters. The mean uncertainty in the metallicities is $\varepsilon[\mathrm{Fe} / \mathrm{H}] \approx 0.10$.

We supplemented these data with the relative abundances of 14 elements in stellar clusters taken from 109 studies published from 1991 through 2015. The elemental abundances in open clusters from all the sources used were determined using high resolution spectra, and were obtained mainly for atmospheres of red giants. Our analysis of the spectra assumed local thermodynamical equilibrium (LTE), but deviations from LTE were taken into account in some studies, primarily for oxygen and magnesium. Most of the studies used Kurucz model stellar atmospheres. Since our aim is to study the chemical evolution of the Galactic thin disk, we restricted our analysis to abundances of elements that are expected to undergo virtually no changes associated with nuclear processes in the envelopes of the studied stars. Such elements include, in particular, the elements ( $\mathrm{O}, \mathrm{Mg}, \mathrm{Si}, \mathrm{Ca}$, and $\mathrm{Ti})$, the iron-peak elements (Fe), and elements produced by slow (Y, Ba, La, Ce, Nd, and Zr) and rapid (Eu) neutron capture; see [18] for a justification of this selection. To these we added two other elements with odd numbers of protons: $\mathrm{Na}$ and $\mathrm{Al}$. The ratios $[\mathrm{el} / \mathrm{Fe}]$ for the clusters were found from the abundances $[\mathrm{el} / \mathrm{H}]$ and $[\mathrm{Fe} / \mathrm{H}]$ contained in the original references, and not the mean metallicities we have presented here.

The mean number of studied stars in a cluster is seven, and the most probable number is four. The abundances were determined using one star in 16 cases; in only 6 clusters was this the only determination, while $[\mathrm{el} / \mathrm{Fe}]$ values are available in other studies in the remaining cases. The maximum number of stars in a cluster analyzed in a single study is 76 (NGC 2243). The elemental abundances for 246 Hyades stars are presented in 15 studies, and for 105 stars in NGC 2682 in 13 studies. A large number of studied stars in a cluster appreciably increases the accuracy with which their mean elemental abundances can be determined. The statistics for the elemental abundance determinations in the open clusters and their uncertainties are presented in Table 1.

We calculated the mean-weighted abundances for clusters with two or more abundance determinations for each of the studied elements. The weighting coefficients were taken to be inversely proportional to the stated uncertainties; as can be seen in Table 1, the corresponding mean values for all elements are in the range $\varepsilon[e l / F e]=(0.06-0.09)$. However, the stated uncertainties for some elements in several studies can sometimes exceed 0.3 dex. These were assigned lower weights, but were included in the catalog unchanged if they were the only available measurement. Fortunately, as was noted above, there are few such uncertain measurements, and they were excluded from consideration if they appreciably influenced the dependences shown by the remaining data. If uncertainties were not indicated, we assumed uncertainties of 0.2 for data published before 2000 and of 0.1 for data published after 2000 .

To estimate the similarity of the abundances of each element obtained in different studies, we analyzed the distribution of the deviations of the published relative abundances for a given cluster from the calculated mean-weighted values; several dozen overlapping values were found for each of the elements studied, apart from cerium and neodymium. All the resulting histograms are fit well 
by normal distributions, indicating that the errors are random. On average, the dispersions are $\sigma[e l / F e]=0.10 \pm 0.01$, with barium and europium displaying the largest dispersions. Thus, the external uncertainties in the relative abundances in clusters are somewhat higher than the values stated in the original papers, on average.

Our resulting catalog contains 346 open clusters with known metallicities, for 272 of which total spatial velocities have been calculated. Table 2 presents a fragment of the full table (catalog), which is accessible in electronic form at the site ftp:// cdsarc.u-strasbg.fr/pub/cats/J/AZh, and contains all the derived parameters for these clusters. The columns of this table give (1) the name of the cluster; (2)-(3) the Galactic coordinates $(l, b)$; (4) the heliocentric distance d; $(5)-(7)$ the coordinates $(\mathrm{x}, \mathrm{y}, \mathrm{z})$ in a right-handed Cartesian coordinate system; (8) the Galactocentric distance $R_{G} ;(9)-(11)$ the three calculated spatial-velocity components $V_{R}, V_{\Theta}, V_{Z}$ in cylindrical coordinates, where $V_{R}$ is directed toward the Galactic anti-center, $V_{\Theta}$ in the direction of the Galactic rotation, and $V_{Z}$ toward the Galactic North pole; (12) the eccentricity of the Galactic orbit e; (13) maximum distance of points in the orbit from the Galactic plane $Z_{\max } ;(14)-(15)$ the apogalactic $R_{a}$ and perigalactic $R_{p}$ distances of the orbit; (16) the cluster age; $(17)-(18)[\mathrm{Fe} / \mathrm{H}]$ and references to the sources for the metallicities; (19)-(32) the relative abundances of 14 elements [el/Fe]; and (33) references to the sources of the chemical compositions. The coding for the $[\mathrm{Fe} / \mathrm{H}]$ and $[\mathrm{el} / \mathrm{Fe}]$ reference numbers are presented in electronic form in the catalog.

For comparison, we considered uniform abundance determinations for the same elements based on high-resolution spectra of 212 field dwarfs and 171 field red giants, since it is such high-luminosity stars that were usually observed in the clusters. All these measurements were obtained by a single team of authors [19]-[22]. We used Kurucz models to determine the elemental abundances in these stars. The uncertainties in the abundances of all elements do not exceed 0.15 dex. We were able to find the distances, proper motions, and radial velocities for all the giants in the new Hipparcos catalog [23. We used these data together with the Galaxy model of Gardner and Flynn [10] to calculate the elements of the Galactic orbits. Since masses for the giants are also available in the literature, we used these to calculate the giant ages in years, using the relation for the main-sequence lifetime for stars with solar metallicity $l g t=10-3.6 l g\left(M / M_{\odot}\right)+l g^{2}\left(M / M_{\odot}\right)$ [24]. We also considered for comparison uniform estimates of the abundances of the same elements based on high-resolution spectra of 221 field Cepheids from [18], located at the same distances as the open clusters. Since the Cepheid elemental abundances were determined by the same authors as those determining the field dwarfs and giants, the uncertainties in $\varepsilon[\mathrm{el} / \mathrm{Fe}]$ do not exceed those for the abundances in these other field stars. See [18] for more detail on the parameters of these comparison stars.

In our current study, we investigated the behavior of the relative abundances of $\alpha$-elements using our compiled catalog; the properties of other elemental abundances will be considered in subsequent papers.

\section{METALLICITY DEPENDENCE OF THE RELATIVE ABUN- DANCES OF $\alpha$ ELEMENTS}

The variation in the relative abundances of some elements $[\mathrm{el} / \mathrm{Fe}]$ with increasing metallicity can be interpreted in terms of the chemical evolution of a stellar-gaseous system only if the overall abundance of heavy elements in the system increases with time, on average; i. e., if the metallicity is a statistical indicator of age. Studies of the metallicity in the Galactic thin disk show that stars of any age display large scatters in their heavy-element contents, hindering identification of a relation between age and metallicity in a subsystem. Many studies have reported an absence of such a relation (see, e.g., [25]-[28]). However, this conclusion has been questioned in other studies, which have suggested that a relation is present, but is blurred by the uncertainties in the stellar ages and the radial migration of stars (see, e.g., [29-[33]).

A complete sample of $14000 \mathrm{~F}-\mathrm{G}$ dwarfs of the thin disk located within $70 \mathrm{pc}$ of the Sun was 
compiled in [34, based on the catalog [27]. Selection effects, systematic effects associated with random errors in the age determinations, and the effects of radial migration of stars were analyzed. It was shown in 34 that, during the first several billion years of the formation of the thin disk, the interstellar material incident on the disk possessed approximately the same heavy-element abundance $(\langle[\mathrm{Fe} / \mathrm{H}]\rangle \approx-0.2)$ and a low degree of homogeneity, but the dispersion in the metallicity $\sigma[\mathrm{Fe} / \mathrm{H}]$ decreased smoothly from $\approx 0.22$ to $\approx 0.13$ with age. However, approximately four to five billion years ago, the mean metallicity began to systematically increase, while preserving the same dispersion. A similar behavior for the age dependence of the metallicity is also displayed by thin-disk stars with uniform spectroscopic iron abundances from the compiled catalog [35], which contains about $900 \mathrm{~F}-\mathrm{G}$ dwarfs and ages estimated from the chromospheric activity of the stars.

It is proposed in [34] that the low star-formation rate in the initial stages of formation of the Galactic thin disk suddenly increased about four to five billion years ago. This means that the metallicity in the thin disk over the past $\sim 5$ billion years can be considered to be related to age, so that the observed differences in the relative abundances of elements in the open clusters compared to the field stars can be interpreted in terms of the chemical evolution of the interstellar matter. This makes it interesting to compare the metallicity dependences of the relative abundances of $\alpha$-elements for the open clusters and the field stars.

According to modern views, $\alpha$-elements are primarily synthesized in the cores of nassive stars $\left(M>10 M_{\odot}\right)$ in late stages of their evolution, and are ejected into the interstellar medium when they explode as Type II supernovae (see, e. g., [34, 37]). Type II supernovae also give rise to some iron-peak elements, but iron-peak elements are produced primarily in Type Ia supernovae, which correspond to the final stage in the evolution of close binary stars with masses $<8 M_{\odot}$ (see, e.g., [38]). Since the evolution times of massive stars do not exceed $\approx 30$ million years and massive Type Ia supernovae occur only after $\approx 1-1.5$ billion years, the relative abundances $[\alpha / \mathrm{Fe}]$ of new generations of stars should decrease as the metallicity increases. The lower the star-forming rate in a stellar-gaseous system, the lower the metallicity at which this decrease begins, and the steeper it is.

Another important property of the abundances of $\alpha$-elements can also be used to analyze the star formation history. According to current views, only oxygen, and possibly magnesium, are produced entirely in massive stars, while the two other $\alpha$-elements we are using are often produced in less massive stars. (Titanium is often not considered an $\alpha$-element, and we do not consider it here.) Theoretical computations show that the output of so-called primary $\alpha$ elements $(\mathrm{O}, \mathrm{Mg})$, synthesized in hydrostatic processes in carbon cores and explosively through $\mathrm{Ne} / \mathrm{C}$ burning in layer sources in pre-supernovae, increases with the mass of the pre-supernova (see, e.g., [39, 40]). Therefore, the observed ratios $[\alpha / \mathrm{Fe}]$ in the atmospheres of new-generation stars will increase as the initial mass function is shifted toward higher masses.

A more trustworthy indicator of the maximum masses of supernovae is the ratio of the primary and secondary $(\mathrm{Si}, \mathrm{Ca}) \alpha$-elements. However, the fact that secondary $\alpha$-elements are also produced in lowermass stars must be taken into account [41. These properties of the $\alpha$-element abundances enable comparison of the maximum masses of supernovae that have enriched clouds producing clusters with various origins, as well as the interstellar medium of the thin disk.

Figure 1 shows the metallicity dependences of the relative abundances of each of the four $\alpha$ elements considered in open clusters (circles) and in field red giants (x's). The relative oxygen abundances for the field giants show a moderate increase with decreasing metallicity, while the slope of the corresponding relation for the open clusters is much lower, although it differs from zero by more than $3 \sigma$ (the correlation coefficients are $r=-0.72 \pm 0.10$ with the probability that the correlation arose by chance being $P_{N} \ll 1 \%$, and $r=-0.41 \pm 0.13$ with $P_{N}<2 \%$, respectively). As a result, the relative oxygen abundances for most of the clusters with metallicities higher than the solar value are approximately the same as those for the field giants; roughly half the lower-metallicity clusters lie below the band occupied by field stars. Simultaneously, a substantial scatter in the $[\mathrm{O} / \mathrm{Fe}]$ values is observed for the clusters, especially for those with metallicities below the solar value.

A similar difference in the slopes is observed for magnesium, but the relative $\mathrm{Mg}$ abundances for 
most of the clusters and field giants coincide at small metallicities. Some clusters have uncharacteristically low or high $[\mathrm{Mg} / \mathrm{Fe}]$ ratios for field giants. At the same time, the overwhelming majority of clusters with higher than solar metallicities lie above the mean values for field giants (i. e., above the corresponding fitted lines).

The dependences for silicon and calcium are very similar for the two types of objects due to the appreciable decrease in the slopes for these secondary $\alpha$-elements for the field stars. For both elements, the scatter of the points in the diagrams is appreciably higher for the clusters than for the field giants. Given that the field giants currently located in the solar vicinity were born at greater Galactocentric distances, comparable to the distances to the clusters (Fig. 4), and the range of ages for the two types of objects are the same (Fig. 3), this suggests that the smaller scatter in the relative abundances of $\alpha$-elements displayed by the field giants indicates that the interstellar medium from which they formed was more uniform than the medium from which the open-cluster stars formed.

Let us now consider another property of all these diagrams. We showed in $[3$, 4 that open clusters can be divided into two groups according to the orbital parameter $\left(Z_{\max }^{2}+4 e^{2}\right)^{1 / 2}$ proposed in [42], with the division being at 0.35 (here, $e$ is the eccentricity of the Galactic orbit and $Z_{\text {max }}$ the maximum distance of the orbit from the Galactic plane in kiloparsec). We referred to clusters with low orbital parameters as "Galactic" and to those with high orbital parameters as "peculiar". The peculiar group also includes clusters whose orbital elements are unknown, but that are located more than $350 \mathrm{pc}$ from the Galactic plane; i. e., they satisfy the criterion above for peculiar clusters. Note that the peculiar group also includes clusters with low orbital parameters but uncharacteristically low metallicities for thin-disk objects, $[\mathrm{Fe} / \mathrm{H}] \approx<-0.2$. However, we did not find published elemental abundances for these clusters. As a result, all the clusters in the sample considered here with high, elongated orbits (i.e., with high orbital parameters) are peculiar, and all the clusters with low, circular orbits are Galactic.

Following Vande Putte etal. [2], we proposed that clusters with high, elongated orbits have an "unusual" origin. In [3, 4, we used the orbital elements calculated in [1, while here we have calculated the orbital elements ourselves, but using another model for the Galaxy; this became possible due to the increasing number of clusters with measured radial velocities. This led to a modest increase in the sizes of the calculated orbits, so that the division of the clusters into the two groups corresponds to the critical value for the orbital parameter $\left(Z_{\max }^{2}+4 e^{2}\right)^{1 / 2}=0.40$ and a distance from the Galactic plane $|z|=400 \mathrm{pc}$. In all the diagrams presented here, the two groups of clusters are denoted using different symbols: peculiar clusters by grey circles and Galactic clusters by circled dots. Our analysis has shown that none of the dispersions of the relative abundances of the $\alpha$ elements display significant differences between the two groups. However, the relative abundances of each of the $\alpha$ elements are higher, on average, for the peculiar clusters than for the higher-metallicity Galactic clusters, which is a consequence of the general negative correlation between $[\mathrm{el} / \mathrm{Fe}]$ and $[\mathrm{Fe} / \mathrm{H}]$. In order to reduce the random errors in the abundances of each element, we averaged the abundances of all four $\alpha$-elements $(\mathrm{O}, \mathrm{Mg}, \mathrm{Si}$, and $\mathrm{Ca}$ ). Since, as follows from Fig. 1, the relative abundances of each of the four $\alpha$-elements for each object display different deviations from the solar values, when aiming to reveal undistorted dependences, the data for the clusters and field stars should be averaged over all four $\alpha$-elements simultaneously. Our analysis shows that the distribution of the deviations of the relative abundances of each $\alpha$-element from the calculated values of $[\alpha / \mathrm{Fe}]$, constructed using 53 clusters with four determinations of the $\alpha$-elements, is described well by a normal law with dispersion $\sigma[\alpha / F e]=0.09 \pm 0.01$, which we take to be an estimate of the uncertainty in this quantity. Although this estimate is comparable to the discrepancies for individual elements indicated in Table 1, the reliability of the mean elemental abundances is generally higher, since each was produced mainly in a single process.

Figure 2 presents the metallicity dependences of the averaged relative abundances of $\alpha$-elements for the open clusters and field red giants and dwarfs. The sequence of field dwarfs and giants, and the corresponding fitted lines, essentially coincide (the fitted line for the dwarfs is not shown, to avoid cluttering the figure). A comparison of Figs. 1 and 2 shows that averaging the four elements for the 
orbits appreciably decreases the scatter in the relative abundances for a fixed metallicity.

It follows from Fig. 2a that the correlations between the relative abundances of the $\alpha$-elements have high significances for both the clusters and field giants $(r=0.59 \pm 0.06$ and $0.88 \pm 0.03$, with $P_{N} \ll 1 \%$ in both cases), with the slope of the dependence for the clusters being significantly lower than that for the field giants (see Fig. 2a), while the scatter of the $[\alpha / \mathrm{Fe}]$ ratios are higher. This trend is due exclusively to the behavior of the clusters with high, elongated orbits, while the Galactic clusters essentially lie in the band occupied by the field stars. However, the scatter of the $[\alpha / \mathrm{Fe}]$ ratios within both groups of clusters are roughly the same. The slope of the dependence for the peculiar clusters is even smaller: $-0.13 \pm 0.04$ (this fitted line is not shown in the figure). The dependences for the clusters and field stars intersect near the solar metallicity and the mean relative abundance of $\alpha$-elements.

Thus, lower-metallicity clusters with high, elongated orbits display lower $[\alpha / \mathrm{Fe}]$ ratios, while the corresponding values in higher-metallicity clusters are slightly higher than those for field stars. For example, 17 of 19 clusters with high, elongated orbits and metallicities $[\mathrm{Fe} / \mathrm{H}]<-0.1$ lie below the fitted line for field stars (note that the abundance of $\alpha$-elements for the cluster NGC 2266, which lies above this line, was determined from data for only one star in one study, and the other cluster lying above the line, Berkeley 21, has been suggested by some authors to have a very low metallicity). In contrast, only 3 of 11 such clusters with high metallicities are located below this line (and those are within 0.03 dex of the line). It is implausible that this came about due to a systematic reduction/enhancement in the abundances of $\alpha$-elements in low/high-metallicity clusters.

The mainly low ratios $[\alpha / \mathrm{Fe}]$ for low-metallicity clusters come about purely due to the low abundances of the primary $\alpha$-elements, as is clearly visible in Fig. 2b, which presents the metallicity dependences of the ratios of the abundances of primary and secondary $\alpha$-elements for clusters and field stars. At low metallicities, the $[\mathrm{O}, \mathrm{Mg} / \mathrm{Si}, \mathrm{Ca}]$ ratios for most clusters are appreciably lower than those for field giants, while the opposite is true at high metallicities. The Galactic clusters also display different ratios of primary to secondary $\alpha$-elements from the field stars, but not from metalrich clusters with high, elongated orbits. As a result, solar values for the ratios of these elements are observed for clusters of any metallicity; i. e., there is essentially no correlation $(r=-0.19 \pm 0.09$ with $P_{N} \approx 17 \%$ ). At the same time, the field giants show a clear, highly significant anti-correlation $\left(r=-0.86 \pm 0.05\right.$ with $\left.P_{N} \ll 1 \%\right)$. The well defined dependence of $[\mathrm{O}, \mathrm{Mg} / \mathrm{Si}, \mathrm{Ca}]$ on $[\mathrm{Fe} / \mathrm{H}]$ for the field giants could come about due to the fact that, with time, the interstellar medium of the thin disk begins to become enriched with secondary $\alpha$-elements produced in Type II and Type Ia supernovae [41, which simultaneously lead to an increase in its metallicity.

Figures $2 \mathrm{c}$ and $3 \mathrm{~d}$ show $[\alpha / \mathrm{Fe}]$ versus $[\mathrm{Fe} / \mathrm{H}]$ for the primary and secondary $\alpha$-elements, respectively. Both correlations for the clusters are highly significant $(r=-0.51 \pm 0.09$ and $-0.41 \pm 0.08$, respectively, with $P_{N} \ll 1 \%$ in both cases). The slopes of the fitted lines for the primary and secondary $\alpha$ elements for the clusters are nearly the same, while those for the field stars differ appreciably. As a result, the sequence of secondary $\alpha$-elements for the clusters and field giants essentially coincide in Fig. 2d. It is striking that, in both diagrams, clusters with low, circular orbits lie mainly in the bands occupied by field stars. As a result, the slopes of the two fitted lines for clusters with high, elongated orbits in Figs. 2c and 3d are still lower. Thus, the differences between clusters with high, elongated orbits and field giants become still larger for primary $\alpha$-elements, and smaller for secondary $\alpha$-elements. Clusters that deviate substantially from the sequence for the field stars are shown in a different font in Fig. 2c. The bold font denotes clusters in which the oxygen and magnesium abundances were determined in several studies, and regular font those in which these abundances were determined in only one study, but using several stars (only the abundances in Be 31 were determined using only one star).

When the deviant clusters delineated using regular font are excluded, the fitted slope for the remaining clusters remains the same, within the uncertainties. Thus, the differences in the slopes of the metallicity dependences of the relative abundances of primary $\alpha$-elements for clusters with high, elongated orbits and field giants are unlikely to be due to systematic errors in the oxygen and 
magnesium abundances of the cluster stars. (Note that the procedure used to test the stability of the fitted lines obtained by excluding deviant points was carried out for all the diagrams in Figs. 1 and 2 , and the fitted slopes for the clusters remained the same within the errors.)

\section{AGE DEPENDENCES OF THE METALLICITY AND RELA- TIVE ABUNDANCES OF $\alpha$ ELEMENTS}

Metallicity. Figure 3a presents age-metallicity diagrams for the open clusters and field red giants. Since it was only possible to calculate a lower limit for the ages of the red giants based on their masses, which are presented in the original references with accuracy to within one figure after the decimal place, these data yielded discrete diagrams. This step was equal to several billion years for giants with high ages, i. e., low masses, while it decreased to tens of millions of years for younger giants. In all, we obtained nine distinct age values. As can be seen in Fig. 3 and Fig. 4, all the age dependences considered deviate appreciably from linear trends. Therefore, when comparing the behavior of the age dependences of the metallicities for open clusters and field giants, it was most convenient to approximate the latter using line segments joining the mean metallicities at the nine age points, while the cluster dependences were approximated using trends obtained from a running average. The mean absolute uncertainties for the cluster ages are presented in the figure, but the uncertainties in the ages are reflected more accurately by their relative uncertainties, which are $\approx 30 \%$, on average 3 .

Figure 3a shows that the behavior of the field red giants is in good agreement with the behavior of the field dwarfs in 34] described above-the systematic decrease in the metallicities before five billion years is obvious, and the dependence becomes flat with further increase in the age. (The one point near 1.7 bilion years that disrupts the smooth behavior of the age dependence of the metallicity for the field giants has a small mean-square error, but represents an average over only five stars, and is unlikely to be reliable.) In addition, a large scatter in the metallicities exceeding the random uncertainties in $[\mathrm{Fe} / \mathrm{H}]$ is observed for both the field giants and dwarfs. Note that the coincidence of the general appearance of the age-metallicity relations for the field red giants and dwarfs suggests that systematic effects associated with uncertainties in the models for the red-giant atmospheres have not led to appreciable distortion of their derived ages. It is also significant that the dwarf ages determined in [34] were obtained both using theoretical isochrones, which are likewise dependent on theoretical atmospheres, and the stars' chromospheric activity.

There is no correlation between age and metallicity for the clusters as a whole $(r=-0.1 \pm 0.3$ with $P_{N} \approx 28 \%$ ), although the mean metallicity varies in a complex fashion with increasing age. In contrast to the age-metallicity dependence for the field stars, which reflects evolutionary variations in the chemical composition of the interstellar medium in the thin disk, this dependence for the clusters is most likely due to differences in the lifetimes of clusters with different orbits [4]. As the figure shows, the overwhelming majority of high-metallicity clusters $([\mathrm{Fe} / \mathrm{H}]>-0.2)$ have low, circular orbits (denoted by circled points in all diagrams); due to their small residual velocities, they remain near the spiral density wave that stimulated their birth over long times, leading to the rapid disruption of such clusters. The bulk of clusters with such orbits $(\approx 90 \%)$ have ages of less than 0.5 billion years. In contrast, almost all the clusters with high ages for which the total velocities have been measured have high, elongated orbits (shown by light filled circles in the diagrams). It is believed that clusters with high, elongated orbits and high metallicities were formed from interstellar material of the thin disk that received a high initial velocity when it was born due to globular clusters intersecting the Galactic plane, while lower-metallicity clusters with similar orbits obtained both their momenta and their low metallicities from giant molecular clouds [4] (see also references therein). As a result, clusters that end up in high, elongated orbits spend a large fraction of their time far from spiral arms, and are not subject to further disruption.

This suggests that, when total-velocity measurements become available for all clusters, old clusters 
will probably end up in high, elongated orbits. Figure 3a shows that a rapid increase in the mean metallicity with increasing age is observed for clusters with ages $<0.3$ billion years. (The behavior of clusters with small ages can be seen in more detail in Fig. 1b of [4], which presents a similar agemetallicity diagram for clusters using a logarithmic age scale.) This is associated with the growth in the fraction of low-metallicity clusters among the youngest clusters. In particular, only one-quarter of clusters younger than 20 million years with known metallicities have metallicities higher than the solar value. At 0.3 billion years, the mean metallicities of the clusters and field giants agree within the uncertainties; further, the mean metallicities of clusters begin to decrease much more rapidly than those of field stars; at two billion years, they have become roughly the same as those of field giants older than $\approx 4.5$ billion years.

The decrease in the mean metallicity of the clusters is associated with a decrease in the fraction of long-lived Galactic high-metallicity clusters with increasing age. The metallicities of clusters older than $\approx 2$ billion years display a very high dispersion due to the absence of a concentration toward some distinct metallicity value, since most high-metallicity clusters whose orbits are located fully in the plane of the Galaxy have already been disrupted. As a result, long-lived clusters with ages of $0.5-4$ billion years have lower metallicities, on average, than do field stars. Among even older clusters, we again note a tendency for an increase in the mean metallicity due to the dominance of clusters with metallicities that are uncharacteristically high for field stars of the same age.

$\alpha$-elements. Figure 3b plots the age versus the relative oxygen abundances for field red giants and open clusters. The mean $[\mathrm{O} / \mathrm{Fe}]$ ratios of the field giants initially increase, but then become constant within the uncertainties after $\approx 4.5$ billion years. This ratio also initially increases for the clusters, but only to ages of roughly two billion years, after which there is even some decrease with age. The sequence of clusters everywhere lies below the points for the field giants, although the two are very close at ages $<2$ billion years. Here also, the slope $s$ of the age dependence of $[\mathrm{O} / \mathrm{Fe}]$ for the clusters is essentially zero $\left(s_{\text {clust }}=0.004 \pm 0.009\right.$ billion years $\left.{ }^{-1}\right)$, while this slope is significantly different from zero for the giants $\left(s_{\text {giant }}=0.013 \pm 0.004\right.$ billion years $\left.^{-1}\right)$.

The age dependence of the relative magnesium abundances in Fig. 3c behave somewhat differently. As in the case with oxygen, the $[\mathrm{Mg} / \mathrm{Fe}]$ ratios for the field giants increase monotonically with age at small ages, but cease to grow after $\approx 4.5$ billion years, while the rapid growth continues only to $\approx 0.5$ billion years in the clusters. In contrast to oxygen, the two lines essentially coincide at large ages; before $\approx 3$ billion years, the mean line for the clusters lies above the analogous line for the field giants, with the difference being outside the uncertainties. The overall slopes for the magnesium dependence for the field giants is significantly greater than zero $\left(s_{\text {giant }}=0.025 \pm 0.003\right.$ billion years $\left.{ }^{-1}\right)$, while the corresponding slope for the clusters is zero within $3 \sigma\left(s_{o c}=0.022 \pm 0.008\right.$ billion years $\left.^{-1}\right)$. The age dependences of the relative abundances of silicon and calcium for the clusters and field giants shown in Figs. 3d and 3e essentially coincide at small ages; further, the sequences for the clusters lies outside the uncertainties in the mean values below. The overall age variations for both objects reproduce the behavior of the other two elements, and both slopes for the clusters are equal to zero within the uncertainties.

Without considering specific origins for the differences in the behaviors of the different $\alpha$-elements, which could be partially associated with both systematic errors and real differences in their abundances, let us consider the age dependence of the relative abundances of the $\alpha$-elements averaged over all four elements. Here also, the fitted slope for the field giants is greater than zero, while the slope for the clusters is zero within the uncertainties $\left(s_{\text {giant }}=0.014 \pm 0.002\right.$ billion years $^{-1}$ and $s_{o c}=0.010 \pm 0.004$ billion years $^{-1}$ ). Figure $3 f$ shows that the dependences for both types of object pass through the coordinate origin; i. e., the current mean relative abundances of the $\alpha$-elements are solar. These relations then grow in roughly the same way until $\approx 2$ billion years, but the growth in the clusters stops at $[\alpha / F e] \approx+0.07$, while it continues in the giants to $\approx 4.5$ billion years, when $[\alpha / F e] \approx+0.13$. The initial increase in $[\alpha / \mathrm{Fe}]$ for the clusters was appreciably slower than for the field giants, as was the case for the increase in the metallicity in Fig. 3a.

Recall that, among the clusters with ages of more than 2 billion years, only two (Collinder 106 
and Collinder 261) have low, circular orbits, while all the remaining old clusters have elongated, high orbits. These clusters probably formed from a mixture of material that had undergone chemical evolution that was different from the interstellar matter of the thin disk, and $[\alpha / \mathrm{Fe}]$ values that differ from those for field stars are often observed. As can be seen in Fig. $3 \mathrm{f}$ and Fig. 4, the decrease in the mean $[\alpha / \mathrm{Fe}]$ ratios with decreasing age arises purely due to the low relative abundances of $\alpha$-elements in clusters with low, circular orbits. The mean $\langle[\alpha / F e]\rangle$ ratios in clusters with elongated, high orbits do not depend on age $\left(s_{o c}=0.005 \pm 0.006\right.$ billion years $\left.{ }^{-1}\right)$.

\section{RELATIONSHIP BETWEEN THE RELATIVE ABUNDANCES OF $\alpha$ ELEMENTS AND LOCATION IN SPACE}

Radial gradients. The abundances of most chemical elements in the Galactic thin disk decrease with Galactocentric distance. These radial gradients can be traced most reliably using Cepheids, which are bright and visible from large distances. The elements of the Galactic orbits of nearby stars can also be used to determine these gradients. In this case, the dependence of the elemental abundances on the maximum or mean orbital radii for the stars can be studied (see, e.g., [43]). The radial metallicity gradient among field stars of the thin disk arises due to the increase in the starformation rate with decreasing distance from the Galactic center 44]. The radial gradients can also be studied using data for open clusters [22, 45]. However, as is pointed out in [2, 4, these include clusters that formed as a result of interactions between the interstellar medium of the thin disk and various massive objects - high-velocity clouds, dwarf galaxies, and globular clusters. This leads to anomalous chemical compositions in these clusters, compared to field stars of the thin disk, as well as high, elongated orbits; therefore, their radial elemental abundance gradients do not reflect the starformation history in the thin disk.

Figure 4a presents the dependence of the metallicities of open clusters on Galactocentric distance $R_{G}$ (six clusters that are located at the largest Galactocentric distances fall outside this diagram). The corresponding dependences for Cepheids and field red giants of the thin disk from [18] and [20]-22 respectively, are shown for comparison. Due to the proximity of the giants, the maximum distances of their orbits from the Galactic center $\left(R_{a}\right)$ were used for their Galactocentric distances. The fitted linear slopes for all of these objects are approximately the same, although the slopes for the clusters are somewhat lower than those for the field stars, even using their true positions (although this difference formally lies within the uncertainties). However, if we use the apogalactic radii of the orbits, the slopes for the clusters become lower, and the radial gradient for the clusters becomes somewhat smaller than the gradient for the field giants $\left(d[\mathrm{Fe} / \mathrm{H}] / d R_{a}=-0.01 \pm 0.01 \mathrm{kpc}^{-1}\right.$ versus $0.05 \pm 0.01 \mathrm{kpc}^{-1}$ ). A smoothed trend of the radius dependence of the metallicity for the clusters obtained using a running average shows that all the slopes are associated with a jump in the metallicity near $9.5 \mathrm{kpc}$, reflecting the existence of two populations of clusters with different properties.

Analysis of the elements of the Galactic orbits presented in [4] showed that the vast majority of all clusters formed within a Galactocentric radius of $\approx 10.5 \mathrm{kpc}$, and within $\approx 180 \mathrm{pc}$ of the Galactic plane. However, due to their high initial velocities, with time, peculiar clusters came to occupy a volume comparable to that occupied by the oldest Galactic subsystems. As a result, peculiar clusters, most having uncharacteristically low-metallicities for field stars of the Galactic disk, have ended up at large Galactocentric distances. Since they formed from interstellar material of the thin disk mixed with various fractions of matter that had undergone different chemical evolution, they could have different abundances of other elements as well.

Although all $\alpha$ elements are ejected into the interstellar medium mainly by the same types of stars, their relative abundances differ somewhat in subsequent generations of stars. Let us consider the behavior of the radial gradients derived for the averaged $\alpha$-elements. Figure $4 \mathrm{~b}$ present the dependences of the relative abundances of $\alpha$-elements in clusters, young Cepheids, and field giants on their Galactocentric distances (five clusters with large $R_{G}$ values fall outside the diagram); $R_{a}$ 
was used here for the field giants. Due to the insufficient number of clusters with known chemical compositions, it is not possible to statistically significantly detect the jump-like variation in the relative abundances at $\approx 9.5 \mathrm{kpc}$ seen in Fig. 4a, although the deficit of clusters in the fourth quadrant of the diagram is consistent with the existence of such a jump.

Figure 4a shows that, as for the field stars, the relative abundances of $\alpha$-elements for the clusters increase with increasing Galactocentric distance. The fitted slopes for the radial gradient of $\alpha$ elements are lower for clusters than for field stars (although they are formally equal within the uncertainties). However, when the apogalactic orbital radii are considered, the difference in the slopes for the clusters and field stars become significant. The gradient $d[\alpha / \mathrm{Fe}] / d R_{G}$ derived using the true positions of the clusters is also smaller than the analogous gradient for field Cepheids $\left(d[\alpha / \mathrm{Fe}] / d R_{G}=\right.$ $0.011 \pm 0.003 \mathrm{kpc}^{-1}$ and $0.018 \pm 0.004 \mathrm{kpc}^{-1}$, respectively). An increase in $[\alpha / \mathrm{Fe}]$ with distance from the Galactic center is easy to understand for the field stars. $\alpha$-elements are produced in the cores of massive stars, which eject them into interstellar space together with some amount of iron when they explode as Type II supernovae. After about a billion years, this matter begins to be enriched in iron-peak elements, which are ejected by less massive Type Ia supernovae, whose progenitors have evolved by this time. Since the star-forming efficiency increases with decreasing distance from the Galactic center, on average, there are fewer metals in more distant stars, leading to higher $[\alpha / \mathrm{Fe}]$ ratios. The Galactocentric positions of the clusters are not determined by the place of their birth, as is the case with field stars, but instead by the velocity they receive when they are born, while the chemical composition is determined by the fraction of low-metallicity matter acquired from the parent high-velocity cloud. As a result, as a rule, clusters that are further from the center have fewer metals.

Vertical gradients. Figures $4 \mathrm{c}$ and $4 \mathrm{~d}$ present the dependences of the metallicity and relative abundances of $\alpha$-elements in clusters on distance from the Galactic plane $(|z|)$, and in field giants on the maximum distance of the orbits from the Galactic plane $\left(Z_{\max }\right)$. As in the plot of $R_{G}$ versus $[\mathrm{Fe} / \mathrm{H}]$, the smoothed trend in the $|z|-[\mathrm{Fe} / \mathrm{H}]$ diagram for clusters displays a sharp drop in the mean metallicity at distances $100 \mathrm{pc}<|z|<200 \mathrm{pc}$, supporting the existence of two populations of clusters. This diagram also shows that the dependences of the metallicity and relative abundances of $\alpha$-elements on distance from the Galactic plane are determined by clusters with high, elongated orbits, as was the case for the dependence on the Galactocentric distances of the clusters. The difference in the slopes of the dependences for the clusters and the field stars is very significant $(0.06 \pm 0.02$ and $0.18 \pm 0.04$, respectively, for Fig. $4 d$ ). If we use $Z_{\max }$ for the clusters, as we did for the field giants, the vertical gradients in the metallicity and relative abundances of $\alpha$-elements for the clusters become still lower (but remain significantly greater than zero). Note that the clusters and field giants behave very similarly in $e-[\mathrm{Fe} / \mathrm{H}]$ and $e-[\alpha / \mathrm{Fe}]$ diagrams ( $e$ is the eccentricity) [3, Fig. 2b]). This is precisely why it is sometimes convenient to consider both of these characteristics of the spatial orbits using a single orbital parameter, $\left(Z_{\max }^{2}+4 e^{2}\right)^{1 / 2}$.

\section{DISCUSSION}

Thus, we see that the primary $\alpha$-elements in open clusters and field stars display different dependences on metallicity, age, Galactocentric distance, and distance from the Galactic plane. Since no origin for the systematic distortions in certain elemental abundances in cluster stars has been found, the detected differences between the behavior of the mean relative abundances of primary $\alpha$-elements in clusters and field stars very likely testify to different chemical evolution histories for the interstellar matter from which these objects formed.

The vast majority of thin-disk field stars are genetically related, since they were formed from the interstellar matter of this Galactic subsystem; all dependences displayed by the relative abundances of the elements they contain reflect the chemical evolution of this subsystem. On the other hand, according to [4], only high-metallicity $([\mathrm{Fe} / \mathrm{H}]>\approx-0.2)$ clusters with low, circular orbits $\left(\left(Z_{\max }^{2}+\right.\right.$ $\left.4 e^{2}\right)^{1 / 2}<0.40$ ), formed from the same material as did the field stars. This possibility is supported 
by the fact that these clusters lie mainly within the band occupied by field stars of the thin disk in all the diagrams.

However, the clusters display appreciably higher metallicities and ratios of primary to secondary $\alpha$-elements (similar to the solar values) than do the field stars, on average. This means that the chemical compositions of the clusters and field stars are somewhat different. Young clusters with low, circular orbits, but with metallicities uncharacteristially low for thin-disk field stars, could plausibly form, for example, from matter from the Magellanic stream that is partially mixed with the interstellar matter in the thin disk. Unfortunately, the red-giant branch has not yet formed in these very distant clusters, and it is not possible to carry out a detailed chemical analysis of the atmospheres of their dwarfs due to their low brightness. Most of these clusters belong to the Perseus stellar complex, as can be seen in Fig. 3c of [45].

Clusters whose orbits are very elongated and extend far from the Galactic plane almost always display different dependences than do field stars. For example, at less than solar metallicities, as a rule, the primary $\alpha$ elements in clusters display lower $[\mathrm{O}, \mathrm{Mg} / \mathrm{Fe}]$ ratios than do field giants and dwarfs of the thin disk (Fig. 2c). All such low-metallicity clusters with measured total velocities are in high, elongated orbits. However, we can see that clusters with high, elongated orbits at more high metallicity have no much lower $[\mathrm{O}, \mathrm{Mg} / \mathrm{Fe}]$ ratios, on average, while field stars display an appreciable decrease in these ratios with increasing $[\mathrm{Fe} / \mathrm{H}]$. As a result, the relative abundances of primary $\alpha$ elements in most of these clusters are higher than those in field giants of the same metallicity.

Note that the metallicity distribution for clusters with high, elongated orbits is bimodal, with two groups separated by a dip near $[\mathrm{Fe} / \mathrm{H}] \approx-0.1$ (Fig. 5a). The parameters of these Gaussians determined from a maximum-likelihood fit show that the probability of erroneously rejecting alternative fits in favor of a sum of two Gaussian curves is $<5 \%$ (see the method in [46]). This bimodality provides appreciable support for the inhomogeneity of this group of clusters. Both metal-poor and metal-rich clusters with high orbital indices are mainly older than 1 billion years (see Fig. 3). The age-metallicity diagram in Fig. 5b shows that both metal-rich and metal-poor clusters with high, elongated orbits display weak $\left(P_{N}>5 \%\right.$ for both) correlations with opposite signs. (Clusters with very low metallicities differing from the mean value for clusters with high, elongated orbits by more than $3 \sigma$ were excluded when obtaining the regression fits.)

Further, it follows from Figs. $4 \mathrm{a}$ and $4 \mathrm{~b}$ that the vast majority of low-metallicity clusters with high, elongated orbits are at distances of $R_{G}>9.5 \mathrm{kpc}$, while all metal-rich clusters are located at smaller Galactocentric distances. Although the slopes and positions of the $[\alpha / \mathrm{Fe}]$ vs. $R_{G}$ dependences for the clusters and field giants are fairly close, their different $[\alpha / \mathrm{Fe}]$ vs. $[\mathrm{Fe} / \mathrm{H}]$ and $[\mathrm{Fe} / \mathrm{H}]$ vs. $R_{G}$ dependences suggest that this is a chance agreement. (Recall that we are supposing that the current Galactocentric positions of peculiar clusters are not associated with their birth places, as is the case for field stars, but rather with their initial velocities, which depend on the velocities of the high-velocity clouds from which they formed.) Finally, clusters with $\left(Z_{\max }^{2}+4 e^{2}\right)^{1 / 2}>0.40$ display appreciable correlations between the distance from the Galactic plane $(|z|)$, on the one hand, and the metallicity and relative abundance of $\alpha$-elements, on the otherthe more non-circular the orbit and the higher the orbit above the Galactic plane, the lower the metallicity and the higher the $[\alpha / \mathrm{Fe}]$ ratio (Figs. $4 \mathrm{c}, 4 \mathrm{~d}$ ). It is possible that, when such clusters were born, they acquired an admixture of matter with some other chemical composition, proportional to the impulse obtained.

In our view, all these facts support the conclusion that low-metallicity clusters with high, elongated orbits contain an admixture of lower-metallicity material with lower relative abundances of primary $\alpha$ elements than the typical values for field stars; i. e., they formed during collisions between highvelocity clouds and the interstellar medium of the thin disk. It appears that the velocity dependence of the metallicity is characteristic for the high-velocity clouds lower-metallicity clouds travel to the Galactic disk from larger distances, and therefore intersect the Galactic plane with higher speeds. Low $[\mathrm{O}, \mathrm{Mg} / \mathrm{Fe}]$ ratios could come about in high-velocity clouds in the presence of such low metallicities if the star-forming rate in the stargas system that gave rise to these clouds is lower than the rate in the thin disk. In this case, Type Ia supernovae, which had already begun to explode in the interstellar 
medium, enriched the material in iron, thereby reducing the $[\alpha / \mathrm{Fe}]$ ratio, although the metallicity itself remained appreciably below the solar value. Moreover, $[\mathrm{O}, \mathrm{Mg} / \mathrm{Fe}]$ ratios in clusters that are lower than in field stars could come about if the mass of Type II supernovae that have enriched the interstellar medium from which the high-velocity clouds were born was lower than in the thin disk.

The appearance of high-metallicity clusters in high, elongated orbits is usually associated with collisions between globular clusters or the cores of decayed dwarf galaxies and the interstellar matter in the thin disk [2] (see also references therein). However, their higher relative abundances of primary $\alpha$ elements compared to field stars of the same metallicity suggests that the birth of these clusters was stimulated by other high-velocity clouds with high abundances of heavy elements and intermediate speeds, which it is believed could form from returning gas in Galactic "fountains" (see, e. g., [47, 48]).

It is supposed that clouds with intermediate velocities are composed of internal Galactic gas, while high-velocity clouds are composed of primordial gas. Observations have indicated enhanced abundances of oxygen (an $\alpha$-element) in such clouds, which can be explained if they form as a result of Type II supernovae. The possibility that these clouds are enriched in material from collapsing stars is also suggested by the fact that enhanced ratios of primary to secondary $\alpha$-elements, compared to field stars, are observed in clusters formed from such clouds. (These ratios are probably substantially lower in field stars due to additional enrichment of the interstellar medium of the thin disk in secondary $\alpha$-elements from Type Ia supernovae [41.)

Note that all the tendencies described here are purely statistical, and clusters that deviate from the generally observed behavior require individual study.

\section{CONCLUSION}

Thus, the distinguishing characteristics of the behavior of the relative abundances of $\alpha$-elements in open clusters support the conclusion that some of the clusters formed during interactions between low metallicity, high-velocity clouds and the interstellar matter of the Galactic thin disk, as was proposed earlier based on analyses of the elements of their Galactic orbits and metallicities. The low ratios of primary $\alpha$-elements in low-metallicity $([\mathrm{Fe} / \mathrm{H}]<-0.1)$ open clusters with high, elongated orbits suggest that these high-velocity clouds were most likely formed from interstellar material in which either the starforming rate was lower or the mass of Type II supernovae was higher than near the Galactic plane. On the other hand, we suggest that the formation of high-metallicity clusters with somewhat less high and elongated orbits is associated with clouds with intermediate velocities that formed from internal gas returning to the disk in Galactic fountains, and that their enhanced relative abundances of primary $\alpha$-elements, compared to field stars, are due to the fact that an appreciable matter in these clouds is from massive Type II supernovae.

Metal-rich $([\mathrm{Fe} / \mathrm{H}]>-0.2)$ clusters with low, circular orbits form a separate group. These are mainly appreciably younger than clusters with high, elongated orbits, and are located at Galactocentric distances comparable to or less than the solar value. Their relative abundances of $\alpha$-elements are the same as those in field giants. These properties support the idea that they are fairly closely and genetically related to matter of the Galactic thin disk. The least metal-rich clusters with circular orbits are young enough that their red-giant branches have not yet formed, and they do not contain stars that can be used for spectroscopic determinations of their elemental abundances. Therefore, it is not possible to elucidate their nature based on their chemical compositions. However, their metallicities, which are uncharacteristically low for Galactic disk stars, suggest the presence of matter from the Magellanic stream in the interstellar matter from which they formed.

Our results and conclusions are valid in the absence of systematic shifts in the elemental abundance determinations, and if our current understanding of the sources of these elements is correct. Our subsequent paper [49] continues our study of the population of open clusters, and presents a statistical

analysis of the relative abundances of neutron-capture elements in clusters of various groups and in field stars of the Galactic thin disk. 


\section{ACKNOWLEDGMENTS}

We thank N.O. Budanova for help in collecting material on the chemical composition of open-cluster stars. This work was supported by the Ministry of Education of the Russian Federation (State contracts No.3.961.2014/K and No.213.01-11/2014- 5, project code 26.63), and the Southern Federal University (grant 213.01-2014/013-VG).

[1] Z. Yu. Wu, X. Zhou, J. Ma, and C.H. Du, Monthly Not. Roy. Astron. Soc. 399, 2146 (2009)

[2] D. Vande Putte, T.P. Garnier, I. Ferreras, R.P. Mignani, and M. Cropper, Monthly Not. Roy. Astron. Soc. 407, 2109 (2010)

[3] M.L. Gozha, T.V. Borkova, and V.A. Marsakov, Astron. Lett. 38, 506 (2012).

[4] M. L. Gozha, V. V. Koval', and V. A. Marsakov, Astron. Lett. 38, 519 (2012).

[5] F. Comeron and J. Torra, Astron. and Astrophys. 261, 94 (1992)

[6] H.R. Jacobson, E.D. Friel, and C.A. Pilachowski, Astron. J. 141, 58 (2011)

[7] G. Carraro and T. Bensby, Monthly Not. Roy. Astron. Soc. 397, 106 (2009)

[8] W.S. Dias, B.S. Alessi, A. Mointinho, and J.R.D. Lépine, Astron. and Astrophys. 389, 871 (2002)

[9] R. Schonrich, J. Binney, and W. Dehnen, Monthly Not. Roy. Astron. Soc. 403, 1829 (2010)

[10] E. Gardner and C. Flynn, Monthly Not. Roy. Astron. Soc. 405, 545 (2010)

[11] U. Heiter, C. Soubiran, M. Netopil, and E. Paunzen, Astron. and Astrophys. 561, 93 (2014)

[12] T. Mishenina, M. Pignatari, G. Carraro, V. Kovtyukh, L. Monaco, S. Korotin, E. Shereta, I. Yegorova, and F. Herwig, Monthly Not. Roy. Astron. Soc. 446, 3651 (2015)

[13] E. Paunzen, U. Heiter, M. Netopil, and C. Soubiran, Astron. and Astrophys. 517, 32 (2010)

[14] H.R. Jacobson and E.D. Friel, Astron. J. 145, 107 (2013)

[15] L. Začs, O. Alksnis, A. Barzdis, A. Laure, F.A. Musaev, A. Bondar, and J. Sperauskas, Monthly Not. Roy. Astron. Soc. 417, 649 (2011)

[16] S. Hekker and J. Melendez, Astron. and Astrophys. 475, 1003 (2007)

[17] I. Akkaya, W.J. Schuster, R. Michel, C. Chavarría-K, A. Moitinho, R. Vázquez, and Y. Karatas, Rev. Mex. Astron. Astrofis. 46, 385 (2010)

[18] V.A. Marsakov, V.V. Koval', V.V. Kovtyukh, and T.V. Mishenina, Astron. Lett. 39, 851 (2013).

[19] T.V. Mishenina, C. Soubiran, V.V. Kovtyukh, and S.A. Korotin, Astron. and Astrophys. 418, $551(2004)$

[20] T.V. Mishenina, O. Bienayme, T.I. Gorbaneva, C. Charbonnel, C. Soubiran, S.A. Korotin, and V.V. Kovtyukh, Astron. and Astrophys. 456, 1109 (2006)

[21] T.V. Mishenina, T.I. Gorbaneva, O. Bienayme, C. Soubiran, V.V. Kovtyukh, and L.F. Orlova, Astron. Rep. 51, 382 (2007). 
[22] T.V. Mishenina, M. Pignatary, S.A. Korotin, C. Soubiran, C. Charbonnel, F.-K. Thielemann, T.I. Gorbaneva, and N.Yu. Basak, Astron. and Astrophys. 552, 128 (2013)

[23] F. van Leeuwen, Astron. and Astrophys. 474, 653 (2007)

[24] V. G. Surdin, Birth of Stars (Editorial URSS, Moscow, 1999) [in Russian].

[25] B. Edvardsson, J. Andersen, B. Gustafsson, D.L. Lambert, P.E. Nissen, and J. Tomkin, Astron. and Astrophys. 275, 101 (1993)

[26] S. Feldzing, J. Holmberg, and J.R. Hurley, Astron. and Astrophys. 377, 911 (2001)

[27] J. Holmberg, B. Nordstrom, and J. Andersen, Astron. and Astrophys. 501, 941 (2009)

[28] M. Bergemann, G.R. Ruchti, A. Serenelli, S. Feltzing, A. Alves-Brito, M. Asplund, T. Bensby, P. Gruiters, U. Heiter, A. Korn, K. Lind, A. Marino, P. Jofre, T. Nordlander, N. Ryde, t al., Astron. and Astrophys. 565, 89 (2014)

[29] F. Pont and L. Eyer, Monthly Not. Roy. Astron. Soc. 351, 487 (2004)

[30] Y. Karatas, S. Bilir, and W.J. Shuster, Monthly Not. Roy. Astron. Soc. 360, 1345 (2005)

[31] H.J. Rocha-Pinto, R.H.O. Rangel, G.F. Porto de Mello, G.A. Bragança, and W.J. Maciel, Astron. and Astrophys. 453, L.9 (2006)

[32] I.N. Reid, E.L. Turner, M.C. Turnbull, M. Mountain, and J.A. Valenti, Astrophys. J. 665, 767 (2007)

[33] M. Haywood, Monthly Not. Roy. Astron. Soc. 388, 1175 (2008)

[34] V.A. Marsakov, V.V. Koval', T.V. Borkova, and M.V. Shapovalov, Astron. Rep. 55, 667 (2011).

[35] T. V. Borkova and V. A. Marsakov, Astron. Rep. 49, 405 (2005).

[36] F.X. Timmes, S.E. Woosley, and T.A. Weaver, Astrophys. J. Suppl. Ser. 98, 617 (1995)

[37] F.-K. Thielemann, K. Nomoto, and Y. Yokio, Astron. and Astrophys. 158, 17 (1986)

[38] F.-K. Thielemann, K. Nomotto, and G. Meyned, Astrophys. J. 460, 408 (1996)

[39] K. Nomoto, K. Iwamoto, N. Nakasato, F.-K. Thielemann, F. Brachwitz, T. Tsujimoto, Y. Kubo, and N. Kishimoto, Nucl. Phys. A 621, 467 (1997)

[40] S.E. Woosley and T.A. Weaver, Astrophys. J. Suppl. Ser. 101, 181 (1995)

[41] I.R. Seitenzahl, F. Ciaraldi-Schoolmann, F.K. Röpke, M. Fink, W. Hillebrandt, M. Kromer, R. Pakmor, A.J. Ruiter, S.A. Sim, and S. Taubenberger, Monthly Not. Roy. Astron. Soc. 429, $1156(2013)$

[42] R.G. Gratton, E. Carretta, R. Claudi, S. Lucatello, and M. Barbieri, Astron. and Astrophys. 404, 187 (2003)

[43] Yu.G. Shevelev and V.A. Marsakov, Astron. Rep. 39, 284 (1995).

[44] V.A. Marsakov and T.V. Borkova, Astron. Lett. 32, 376 (2006).

[45] M.L. Gozha and V.A. Marsakov, Astron. Lett. 39, 171 (2013).

[46] B.R. Martin, Statistics for Physicists (London-N. Y.: Acad. Press, 1971) 
[47] J.N. Bregman, Astrophys. J. 236, 577 (1980)

[48] F. Fraternali, A. Marasco, L. Armillotta, and F. Marinacci, Monthly Not. Roy. Astron. Soc. 447, L.70 (2015)

[49] V.A. Marsakov, M.L. Gozha, V.V. Koval', and L.V. Shpigel', Astron. Rep. 60, 61 (2016). 

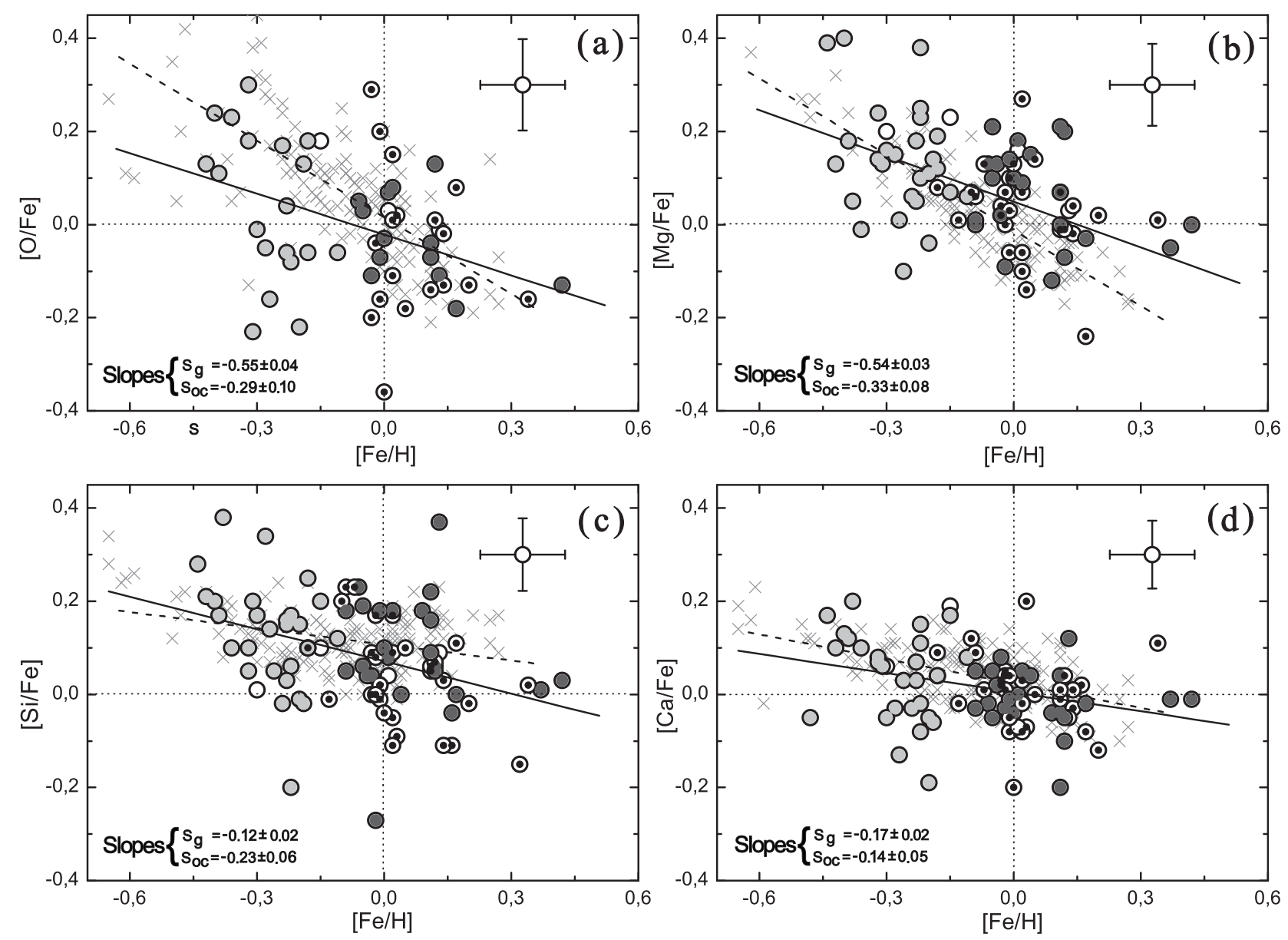

Figure 1: Metallicity dependences of the relative abundances of four $\alpha$ elements in open clusters (circles) and field red giants (x's). The circled dots represent clusters with low, circular orbits (orbital indices $\left(Z_{\max }^{2}+4 e^{2}\right)^{1 / 2}<0.40$ and $\left.|z|<400 \mathrm{pc}\right)$. The light and dark gray filled circles represent low-metallicity $([\mathrm{Fe} / \mathrm{H}]<-0.1)$ and high-metallicity clusters, respectively,with high, elongated orbits. The hollow circles represent unclassified clusters. The solid lines show the linear regression fits for the clusters, and the dashed lines the corresponding fits for the giants. The slopes of the fitted lines and their uncertainties are indicated. The circle with bars shows the characteristic uncertainties for all the elements. The dotted horizontal and vertical lines pass through the solar values for the metallicities and $[\mathrm{el} / \mathrm{Fe}]$. 

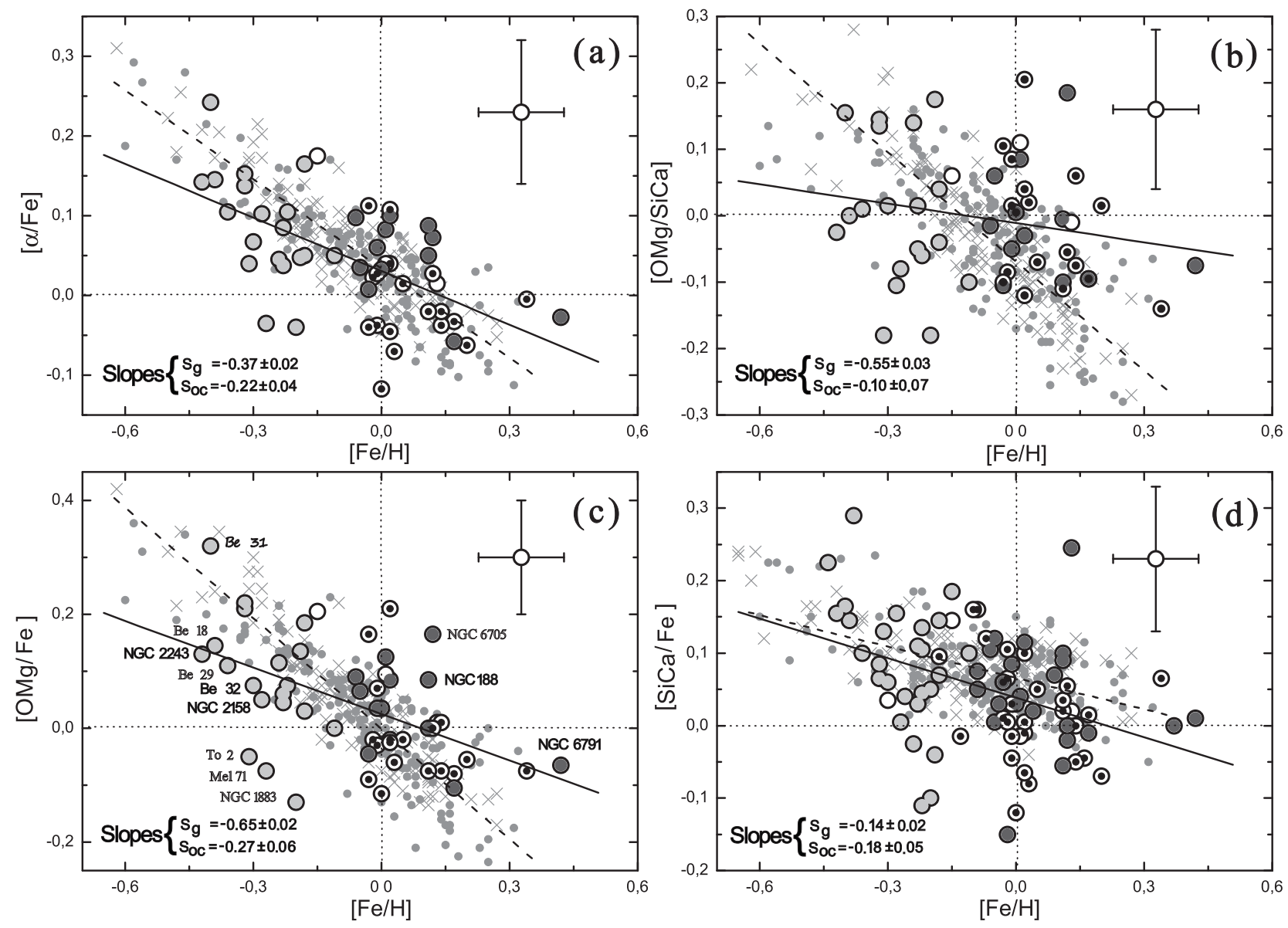

Figure 2: Dependences of the (a) mean relative abundances of $\alpha$ elements, (b) the ratio of the abundances of the primary and secondary $\alpha$-elements, and the relative abundances of the (c) primary and (d) secondary $\alpha$-elements on metallicity, for open clusters (circles), field red giants (x's), and field dwarfs (points). The notation is the same as in Fig. 1. Clusters with high, elongated orbits that have large deviations from the plane occupied by the field giants are indicated in panel (c); the abundances for those in bold font have been determined in several studies, for those in normal font in a single study using several stars, and for those in italic font using data for a single star. 

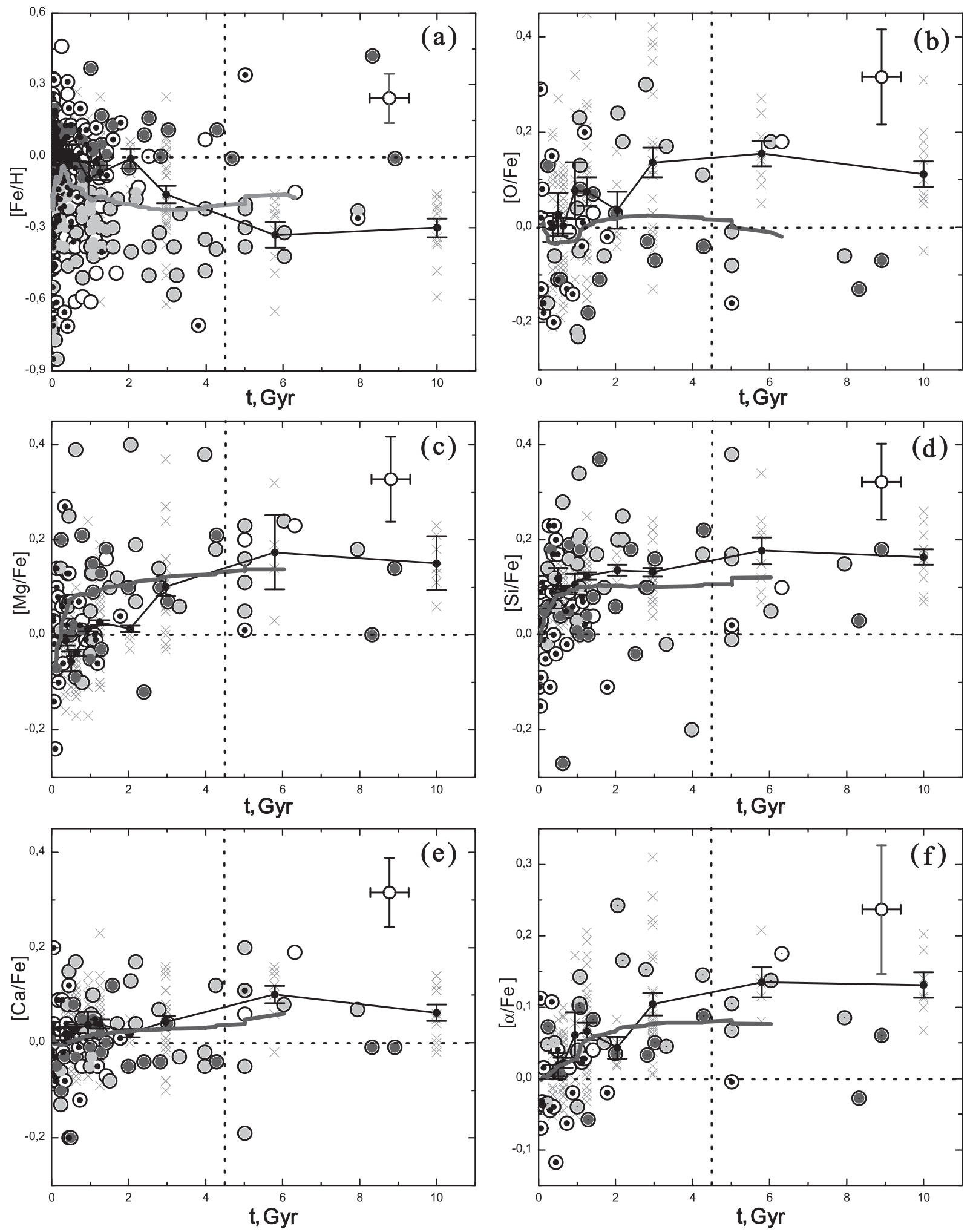

Figure 3: Age dependences of the (a) metallicity, (b)-(e) relative abundances of four $\alpha$-elements, and (f) mean relative abundances of the $\alpha$-elements for open clusters (circles) and red giants of the field (x's). The smooth curves show fitted smoothed trends obtained from a running average of the age dependences of the clusters; the segmented curves show the mean metallicities and $\langle[\alpha / \mathrm{Fe}]\rangle$ ratios in nine narrow age bands for field giants joined by line segments; the bars denote the uncertainty in the mean values for the giants. The remaining notation is as in Fig. 1. 

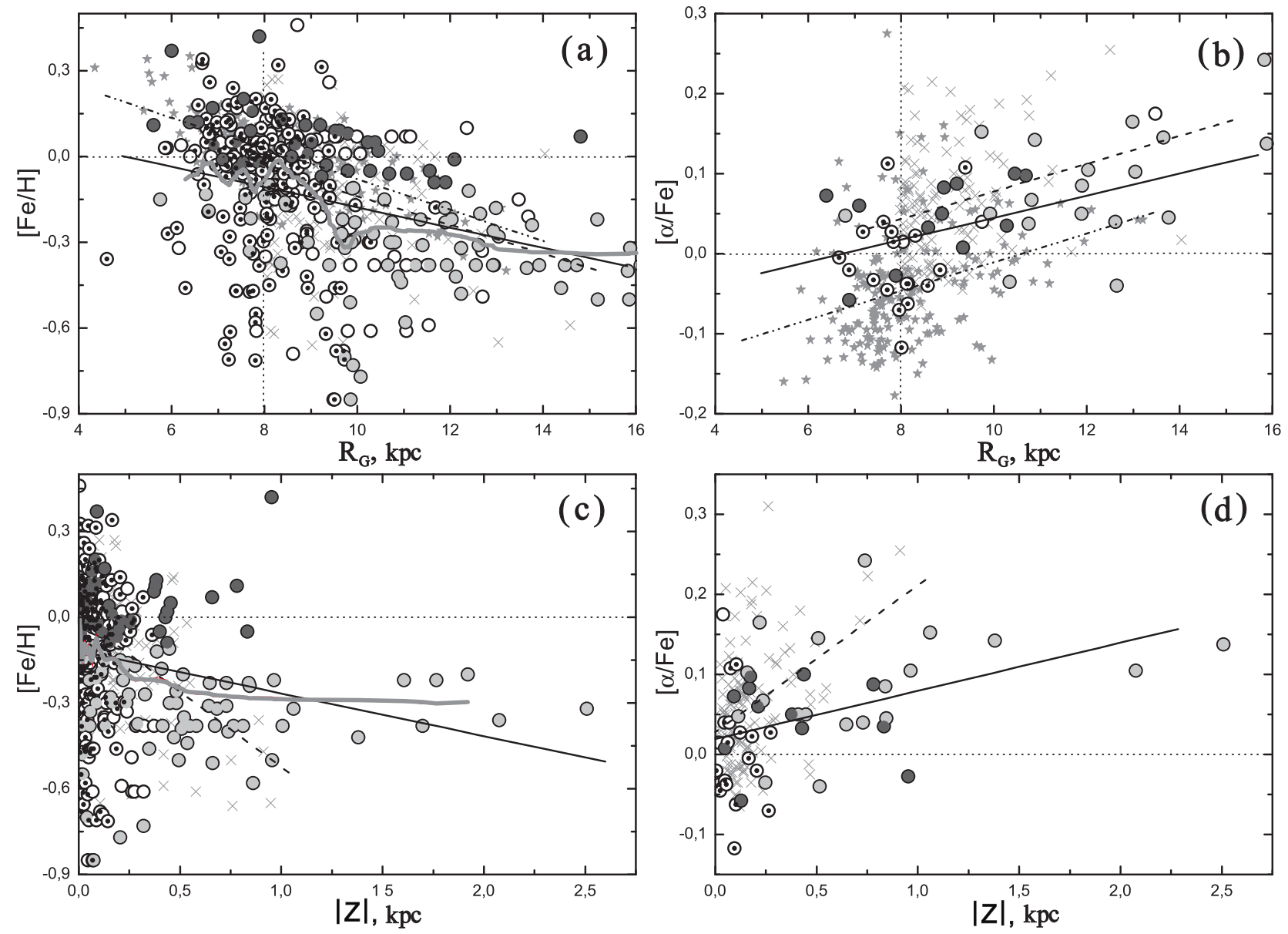

Figure 4: Dependences of the metallicity and mean relative abundances of $\alpha$ elements on $(a, b)$ position in the Galaxy and (c, d) distance from the Galactic plane for open clusters (circles), field red giants (x's), and field Cepheids (stars). In view of their closeness to the Sun, the apogalactic radii of the orbits and maximum distances of the orbits from the Galactic plane are used for the giants. The thick gray line shows the fit for the clusters, the thin dashed line the fit for the field giants, and the thin dotted line the fit for the field Cepheids. The smooth curve is a smoothed trend with a running average for the open clusters. The remaining notation is as in Fig. 1. 

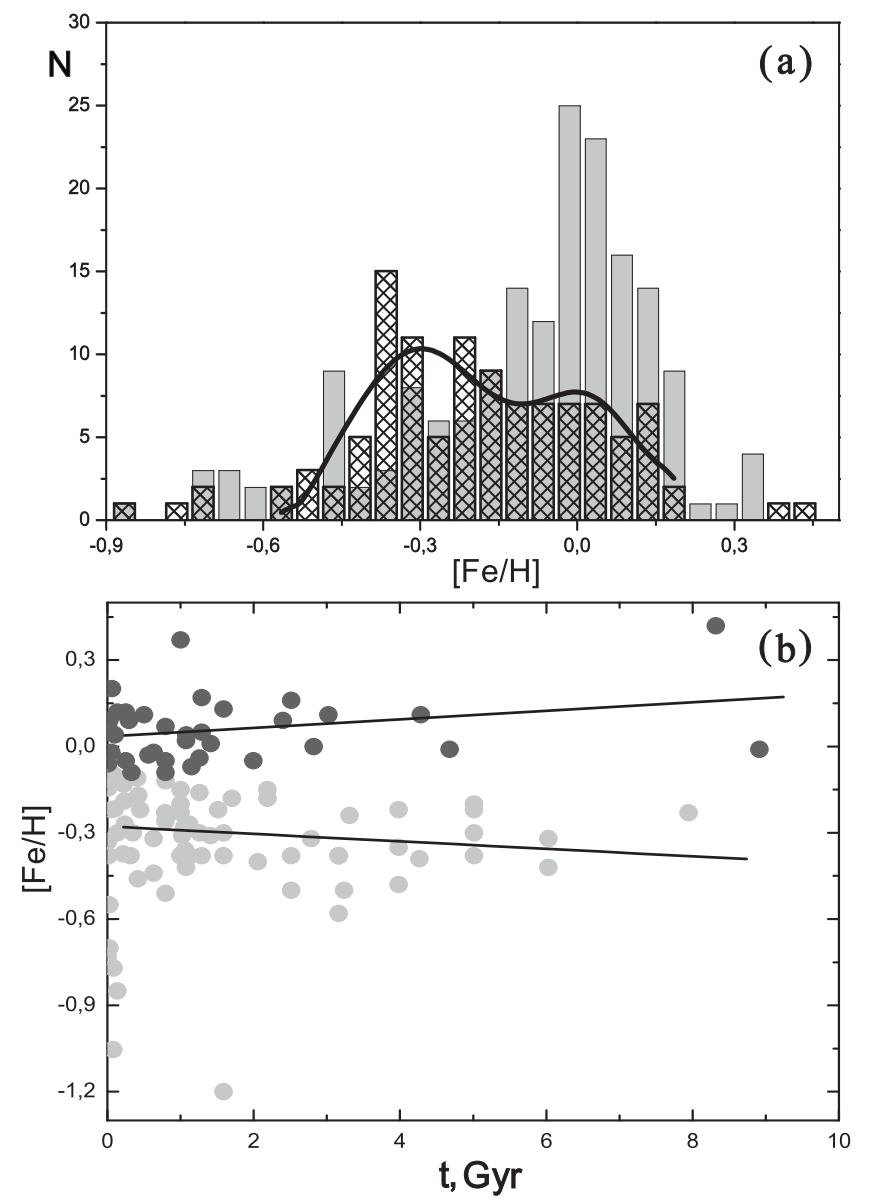

Figure 5: (a) Metallicity distributions for open clusters with low, circular orbits (gray) and with high, elongated orbits (hatched). (b) Age-metallicity dependence for clusters with high, elongated orbits. The curve in (a) is the result of fitting the distribution for high, elongated orbits using a sum of two Gaussian curves. The lines in (b) show linear fits for high-metallicity $([\mathrm{Fe} / \mathrm{H}]>-0.1)$ and low-metallicity clusters. The remaining notation is as in Fig. 1. 
Table 1: Statistics of the elemental abundances in the open clusters and their uncertainties

\begin{tabular}{l|c|c|c|c|c}
\hline \hline & & $\begin{array}{l}\text { Number of } \\
\text { Element }\end{array}$ & $\begin{array}{l}\text { Dispersion } \\
\text { of the } \\
\text { stated } \\
\text { uncertainty } \\
\text { uncertainty }\end{array}$ & $\begin{array}{l}\text { Number of in- } \\
\text { tersecting deter- } \\
\text { minations }\end{array}$ & $\begin{array}{l}\text { Dispersion of } \\
\text { the calculated } \\
\text { mean }\end{array}$ \\
\hline $\mathrm{O}$ & 54 & 0.07 & 0.04 & 56 & 0.09 \\
$\mathrm{Na}$ & 74 & 0.07 & 0.05 & 80 & 0.11 \\
$\mathrm{Mg}$ & 81 & 0.07 & 0.06 & 83 & 0.10 \\
$\mathrm{Al}$ & 71 & 0.07 & 0.07 & 65 & 0.11 \\
$\mathrm{Si}$ & 86 & 0.06 & 0.05 & 100 & 0.10 \\
$\mathrm{Ca}$ & 85 & 0.07 & 0.07 & 109 & 0.09 \\
$\mathrm{Ti}$ & 86 & 0.06 & 0.04 & 93 & 0.09 \\
$\mathrm{Y}$ & 59 & 0.07 & 0.05 & 40 & 0.10 \\
$\mathrm{Zr}$ & 52 & 0.09 & 0.09 & 55 & 0.11 \\
$\mathrm{Ba}$ & 73 & 0.08 & 0.05 & 83 & 0.12 \\
$\mathrm{La}$ & 55 & 0.06 & 0.05 & 41 & 0.04 \\
$\mathrm{Ce}$ & 38 & 0.06 & 0.04 & 12 & 0.09 \\
$\mathrm{Nd}$ & 29 & 0.07 & 0.06 & 9 & 0.12 \\
$\mathrm{Eu}$ & 47 & 0.07 & 0.06 & 26 & \\
\hline
\end{tabular}


Table 2: Elemental abundances and parameters of the Galactic orbits in open clusters (fragment)

\begin{tabular}{|c|c|c|c|c|c|c|c|c|c|c|c|}
\hline Name & $\begin{array}{l}l, \\
\operatorname{deg}\end{array}$ & $\begin{array}{l}b, \\
\text { deg }\end{array}$ & $d, \mathrm{pc}$ & $x, \mathrm{pc}$ & $y, \mathrm{pc}$ & $z, \mathrm{pc}$ & $\begin{array}{l}R_{G} \\
\mathrm{kpc}\end{array}$ & $\begin{array}{l}V_{R}, \\
\mathrm{~km} / \mathrm{s}\end{array}$ & $\begin{array}{l}V_{\Theta}, \\
\mathrm{km} / \mathrm{s}\end{array}$ & $\begin{array}{l}V_{Z}, \\
\mathrm{~km} / \mathrm{s}\end{array}$ & $e$ \\
\hline NGC 6583 & 9.2825 & -2.5336 & 2040 & 2011 & 329 & -90 & 6.00 & -23.8 & 139.9 & 51.5 & 0.322 \\
\hline NGC 6791 & 69.9585 & 10.9039 & 5035 & 1694 & 4645 & 952 & 7.89 & 8.9 & 189.7 & -34.1 & 0.115 \\
\hline NGC 7789 & 115.5319 & -5.3849 & 1795 & -770 & 1613 & -168 & 8.92 & 16.7 & 172.3 & 1.2 & 0.222 \\
\hline NGC 752 & 137.1251 & -23.2541 & 457 & -308 & 286 & -180 & 8.31 & 9.7 & 213.8 & -12.8 & 0.047 \\
\hline NGC 2099 & 177.6354 & 3.0915 & 1383 & -1380 & 57 & 75 & 9.38 & 0.6 & 189.6 & -1.8 & 0.150 \\
\hline Name & $\begin{array}{l}Z_{\max } \\
\mathrm{kpc}\end{array}$ & $\begin{array}{l}R_{a} \\
\mathrm{kpc}\end{array}$ & $\begin{array}{l}R_{p}, \\
\mathrm{kpc}\end{array}$ & age & {$[\mathrm{Fe} / \mathrm{H}]$} & $\begin{array}{l}\text { Ref. } \\
\text { for } \\
{[\mathrm{Fe} / \mathrm{H}]}\end{array}$ & {$[\mathrm{O} / \mathrm{Fe}]$} & {$[\mathrm{Na} / \mathrm{Fe}]$} & {$[\mathrm{Mg} / \mathrm{Fe}]$} & {$[\mathrm{Al} / \mathrm{Fe}]$} & {$[\mathrm{Si} / \mathrm{Fe}]$} \\
\hline NGC 6583 & 0.825 & 6.58 & 3.37 & 1.000 & 0.37 & 50 & & & -0.05 & 0.11 & 0.01 \\
\hline NGC 6791 & 1.310 & 8.30 & 6.58 & 8.318 & 0.42 & 50 & -0.13 & 0.11 & 0.00 & -0.06 & 0.03 \\
\hline NGC 7789 & 0.172 & 9.46 & 6.02 & 1.413 & 0.01 & 50 & 0.13 & 0.07 & 0.18 & 0.08 & 0.00 \\
\hline NGC 752 & 0.293 & 8.90 & 8.11 & 1.122 & -0.02 & 50 & -0.04 & 0.08 & 0.00 & 0.08 & 0.08 \\
\hline NGC 2099 & 0.082 & 9.88 & 7.30 & 0.347 & 0.02 & 50 & 0.15 & 0.09 & 0.27 & -0.06 & 0.09 \\
\hline Name & {$[\mathrm{Ca} / \mathrm{Fe}]$} & {$[\mathrm{Ti} / \mathrm{Fe}]$} & {$[\mathrm{Y} / \mathrm{Fe}]$} & {$[\mathrm{Zr} / \mathrm{Fe}]$} & {$[\mathrm{Ba} / \mathrm{Fe}]$} & {$[\mathrm{La} / \mathrm{Fe}]$} & {$[\mathrm{Ce} / \mathrm{Fe}]$} & {$[\mathrm{Nd} / \mathrm{Fe}]$} & {$[\mathrm{Eu} / \mathrm{Fe}]$} & $\begin{array}{l}\text { Ref. } \\
\text { for } \\
{[\mathrm{el} / \mathrm{Fe}]}\end{array}$ & \\
\hline NGC 6583 & -0.01 & -0.01 & -0.19 & & -0.05 & & & & & 67,13 & \\
\hline NGC 6791 & -0.01 & 0.01 & 0.03 & & -0.05 & & & & -0.17 & $5,17,20,24,47,78$ & \\
\hline NGC 7789 & 0.00 & -0.04 & 0.10 & 0.12 & 0.45 & 0.11 & 0.09 & 0.17 & 0.02 & $61,81,106$ & \\
\hline NGC 752 & 0.05 & -0.04 & -0.01 & 0.06 & 0.38 & 0.12 & 0.13 & 0.16 & 0.06 & $4,22,31,65,88$ & \\
\hline NGC 2099 & -0.08 & -0.08 & -0.07 & & 0.57 & 0.09 & & 0.27 & & 81 & \\
\hline
\end{tabular}

